\title{
Capturing temporal heterogeneity in soil nitrous oxide fluxes with a robust and low-cost automated chamber apparatus
}

Nathaniel C. Lawrence and Steven J. Hall

Correspondence to: Steven J. Hall (stevenjh@iastate.edu)

The copyright of individual parts of the supplement might differ from the CC BY 4.0 License. 
Table S1: Table with the list of primary parts needed to construct the autochambers and control system described in the main manuscript.

\begin{tabular}{|c|c|c|c|c|c|c|c|c|}
\hline Part Name & Purpose & Supplier & $\begin{array}{l}\text { Company } \\
\text { Location }\end{array}$ & Part \# & $\begin{array}{l}\text { Total Cost } \\
\text { (for } 9 \\
\text { chambers) }\end{array}$ & Chamber & $\begin{array}{l}\text { Instrument } \\
\text { Shed }\end{array}$ & Notes \\
\hline $\begin{array}{l}2.54 \mathrm{~cm} \times 7.62 \mathrm{~cm} \\
(1 " \times 3 " \text { nominal) } \\
\text { HDPE bar }\end{array}$ & Chamber base (Fig3a) & $\begin{array}{l}\text { McMaster- } \\
\text { Carr Supply } \\
\text { Company }\end{array}$ & $\begin{array}{l}\text { Elmhurst, IL, } \\
\text { USA }\end{array}$ & $8671 \mathrm{~K} 63$ & 254 & $\mathrm{x}$ & & \\
\hline $\begin{array}{l}\text { Custom Aluminum } \\
\text { L-Brackets }\end{array}$ & Mount lid to frame (Fig3b) & $\begin{array}{l}\text { W. W. } \\
\text { Grainger Inc. }\end{array}$ & $\begin{array}{l}\text { Lake Forest, } \\
\text { IL, USA }\end{array}$ & 2EYW8 & 52 & $\mathrm{x}$ & & $\begin{array}{l}\text { Subsequent } \\
\text { machining } \\
\text { required }\end{array}$ \\
\hline Spherical Rod Ends & Attach lid pivot point to frame (Fig3b) & $\begin{array}{l}\text { McMaster- } \\
\text { Carr Supply } \\
\text { Company }\end{array}$ & $\begin{array}{l}\text { Elmhurst, IL, } \\
\text { USA }\end{array}$ & $60645 \mathrm{~K} 13$ & 61 & $\mathrm{x}$ & & \\
\hline $\begin{array}{l}0.64 \mathrm{~cm} \text { threaded } \\
\text { rod }\end{array}$ & Pivot point/structure for lid (Fig3c) & $\begin{array}{l}\text { McMaster- } \\
\text { Carr Supply } \\
\text { Company }\end{array}$ & $\begin{array}{l}\text { Elmhurst, IL, } \\
\text { USA }\end{array}$ & 98804A112 & 89 & $\mathrm{x}$ & & \\
\hline $\begin{array}{l}0.95 \mathrm{~cm} \text { stainless } \\
\text { tubing }\end{array}$ & Lid structure (Fig3, 1-5) & $\begin{array}{l}\text { W. W. } \\
\text { Grainger Inc. }\end{array}$ & $\begin{array}{l}\text { Lake Forest, } \\
\text { IL, USA }\end{array}$ & 3ADE9 & 209 & $\mathrm{x}$ & & \\
\hline $\begin{array}{l}\text { Aluminum angle } \\
\text { brackets (lid) }\end{array}$ & Fig3, red circles & $\begin{array}{l}\text { W. W. } \\
\text { Grainger Inc. }\end{array}$ & $\begin{array}{l}\text { Lake Forest, } \\
\text { IL, USA }\end{array}$ & 2EYN4 & 7 & $\mathrm{x}$ & & \\
\hline $\begin{array}{l}1.27 \mathrm{~cm} \text { stainless } \\
\text { tubing }\end{array}$ & $\begin{array}{l}\text { Provide additional rigidity around the } \\
\text { threaded rod }\end{array}$ & $\begin{array}{l}\text { W. W. } \\
\text { Grainger Inc. }\end{array}$ & $\begin{array}{l}\text { Lake Forest, } \\
\text { IL, USA }\end{array}$ & 4VMR6 & 50 & $\mathrm{x}$ & & \\
\hline $\begin{array}{l}5.98 \times 2.04 \mathrm{~cm} \\
\text { HDPE bar }\end{array}$ & $\begin{array}{l}\text { Connect piston and threaded rod } \\
\text { (Fig3d, yellow circle) }\end{array}$ & $\begin{array}{l}\text { McMaster- } \\
\text { Carr Supply } \\
\text { Company }\end{array}$ & $\begin{array}{l}\text { Elmhurst, IL, } \\
\text { USA }\end{array}$ & $8671 \mathrm{~K} 38$ & 253 & $\mathrm{x}$ & & \\
\hline Cylinder Rod Piston & Move lid up/down & $\begin{array}{l}\text { Clippard } \\
\text { Instrument } \\
\text { Laboratory } \\
\text { Inc }\end{array}$ & $\begin{array}{l}\text { Cincinnati, } \\
\text { OH, USA }\end{array}$ & UDR-17-6 & 333 & $\mathrm{x}$ & & \\
\hline $\begin{array}{l}\text { Feet to connect } \\
\text { piston to base }\end{array}$ & Move lid up/down & $\begin{array}{l}\text { Clippard } \\
\text { Instrument } \\
\text { Laboratory } \\
\text { Inc }\end{array}$ & $\begin{array}{l}\text { Cincinnati, } \\
\mathrm{OH}, \mathrm{USA}\end{array}$ & CB-1795 & 32 & $\mathrm{x}$ & & \\
\hline $\begin{array}{l}\text { Piston Spherical } \\
\text { Rod End }\end{array}$ & Move lid up/down & $\begin{array}{l}\text { Clippard } \\
\text { Instrument } \\
\text { Laboratory } \\
\text { Inc }\end{array}$ & $\begin{array}{l}\text { Cincinnati, } \\
\mathrm{OH}, \mathrm{USA}\end{array}$ & RE-1785 & 76 & $\mathrm{x}$ & & \\
\hline
\end{tabular}




\begin{tabular}{|c|c|c|c|c|c|c|c|c|}
\hline Part Name & Purpose & Supplier & $\begin{array}{l}\text { Company } \\
\text { Location }\end{array}$ & Part \# & $\begin{array}{l}\text { Total Cost } \\
\text { (for } 9 \\
\text { chambers) }\end{array}$ & Chamber & $\begin{array}{l}\text { Instrument } \\
\text { Shed }\end{array}$ & Notes \\
\hline $\begin{array}{l}1.27 \mathrm{~cm} \mathrm{HDPE} \\
\text { Sheet (lid) }\end{array}$ & Chamber lid (under red circles, Fig3) & $\begin{array}{l}\text { McMaster- } \\
\text { Carr Supply } \\
\text { Company }\end{array}$ & $\begin{array}{l}\text { Elmhurst, IL, } \\
\text { USA }\end{array}$ & $8619 \mathrm{~K} 477$ & 246 & $\mathrm{x}$ & & \\
\hline $\begin{array}{l}\text { Custom Inlet Tube } \\
\text { Barstock }\end{array}$ & Control inlet flow; Fig2a & $\begin{array}{l}\text { W. W. } \\
\text { Grainger Inc. }\end{array}$ & $\begin{array}{l}\text { Lake Forest, } \\
\text { IL, USA }\end{array}$ & 2EYK7 & 77 & $\mathrm{x}$ & & $\begin{array}{l}\text { Subsequent } \\
\text { machining } \\
\text { required }\end{array}$ \\
\hline $\begin{array}{l}10.16 \mathrm{~cm} \text { dia. PVC } \\
\text { tube }\end{array}$ & Protective cover for inlet tube & $\begin{array}{l}\text { Local } \\
\text { Hardware } \\
\text { Supply }\end{array}$ & & & 17 & $\mathrm{x}$ & & \\
\hline $\begin{array}{l}0.64 \mathrm{~cm} \text { HDPE } \\
\text { Sheet (cap) }\end{array}$ & Protective cover for inlet tube & $\begin{array}{l}\text { McMaster- } \\
\text { Carr Supply } \\
\text { Company }\end{array}$ & $\begin{array}{l}\text { Elmhurst, IL, } \\
\text { USA }\end{array}$ & $8619 \mathrm{~K} 467$ & 80 & $\mathrm{x}$ & & \\
\hline $1 \mathrm{~cm}$ spacers & $\begin{array}{l}\text { Elevate protective cover for inlet } \\
\text { above the main chamber lid to enable } \\
\text { gas movement }\end{array}$ & $\begin{array}{l}\text { McMaster- } \\
\text { Carr Supply } \\
\text { Company }\end{array}$ & $\begin{array}{l}\text { Elmhurst, IL, } \\
\text { USA }\end{array}$ & 92510A624 & 36 & $\mathrm{x}$ & & \\
\hline $\begin{array}{l}2.54 \mathrm{~cm} \text { D-Shaped } \\
\text { Rubber Seal }\end{array}$ & Seal lid to collar in soil & $\begin{array}{l}\text { McMaster- } \\
\text { Carr Supply } \\
\text { Company }\end{array}$ & $\begin{array}{l}\text { Elmhurst, IL, } \\
\text { USA }\end{array}$ & 1129A987 & 225 & $\mathrm{x}$ & & \\
\hline $\begin{array}{l}\text { Slotted Steel } \\
\text { Contstruction Strut }\end{array}$ & $\begin{array}{l}\text { Provide additional mass at front of lid } \\
\text { to ensure tight seal of lid on collar }\end{array}$ & $\begin{array}{l}\text { Local } \\
\text { Hardware } \\
\text { Supply }\end{array}$ & & & 117 & $\mathrm{x}$ & & \\
\hline $\begin{array}{l}6.4 \mathrm{~mm}(1 / 4 " \\
\text { nominal }) \mathrm{OD} \\
\text { polyethylene tubing }\end{array}$ & $\begin{array}{l}\text { Chamber outlet gas sampler (mounted } \\
\text { inside chamber lid); perforate at } \\
\text { regular intervals }\end{array}$ & $\begin{array}{l}\text { Cole-Parmer } \\
\text { Instrument } \\
\text { Company }\end{array}$ & $\begin{array}{l}\text { Vernon Hills, } \\
\text { IL, USA }\end{array}$ & $\begin{array}{l}\text { EW- } \\
06490-15\end{array}$ & 72 & $\mathrm{x}$ & & \\
\hline Stainless Eyebolt & $\begin{array}{l}\text { Suspend perforated chamber outlet } \\
\text { manifold sampling tube below the lid }\end{array}$ & $\begin{array}{l}\text { W. W. } \\
\text { Grainger Inc. }\end{array}$ & $\begin{array}{l}\text { Lake Forest, } \\
\text { IL, USA }\end{array}$ & 5ZE79 & 27 & $\mathrm{x}$ & & \\
\hline $\begin{array}{l}0.64 \mathrm{~cm} \text { brass tube } \\
\text { union fitting }\end{array}$ & $\begin{array}{l}\text { Connect } 1 / 4 " \text { nominal gas tubing } \\
\text { lengths }\end{array}$ & $\begin{array}{l}\text { Swagelok } \\
\text { Company }\end{array}$ & $\begin{array}{l}\text { Solon, OH, } \\
\text { USA }\end{array}$ & B-400-6 & 270 & $\mathrm{x}$ & & \\
\hline $\begin{array}{l}0.64 \mathrm{~cm} \text { bulkhead } \\
\text { union }\end{array}$ & $\begin{array}{l}\text { Connect gas transport tubing outside } \\
\text { the chamber to perforated sampling } \\
\text { tubing inside the chamber }\end{array}$ & $\begin{array}{l}\text { Swagelok } \\
\text { Company }\end{array}$ & $\begin{array}{l}\text { Solon, } \mathrm{OH}, \\
\text { USA }\end{array}$ & B-400-61 & 63 & $\mathrm{x}$ & & \\
\hline $0.64 \mathrm{~cm}$ union tee & $\begin{array}{l}\text { Connect gas transport tubing outside } \\
\text { the chamber to perforated sampling } \\
\text { tubing inside the chamber }\end{array}$ & $\begin{array}{l}\text { Swagelok } \\
\text { Company }\end{array}$ & $\begin{array}{l}\text { Solon, } \mathrm{OH}, \\
\text { USA }\end{array}$ & B-400-3 & 90 & $\mathrm{x}$ & & \\
\hline $\begin{array}{l}30.48 \mathrm{~cm} \mathrm{PVC} \\
\text { collars }\end{array}$ & Collar for inserting into the soil & $\begin{array}{l}\text { Local } \\
\text { Hardware } \\
\text { Supply }\end{array}$ & & & 100 & $\mathrm{x}$ & & \\
\hline
\end{tabular}




\begin{tabular}{|c|c|c|c|c|c|c|c|c|}
\hline Part Name & Purpose & Supplier & $\begin{array}{l}\text { Company } \\
\text { Location }\end{array}$ & Part \# & $\begin{array}{l}\text { Total Cost } \\
\text { (for } 9 \\
\text { chambers) }\end{array}$ & Chamber & $\begin{array}{l}\text { Instrument } \\
\text { Shed }\end{array}$ & Notes \\
\hline $\begin{array}{l}\text { Pall } 1 \mu \mathrm{m} \text { filters } \\
(.32 \mathrm{~cm} \text { MNPT })\end{array}$ & $\begin{array}{l}\text { Prevent water / debris from entering } \\
\text { gas sampling tubes }\end{array}$ & $\begin{array}{l}\text { VWR } \\
\text { International }\end{array}$ & $\begin{array}{l}\text { Radnor, PA, } \\
\text { USA }\end{array}$ & $16003-654$ & 828 & $\mathrm{x}$ & $\mathrm{x}$ & \\
\hline $\begin{array}{l}0.32 \text { FNPT } \times 0.64 \\
\text { tube fitting }\end{array}$ & Connect filters to tubing & $\begin{array}{l}\text { Swagelok } \\
\text { Company }\end{array}$ & $\begin{array}{l}\text { Solon, OH, } \\
\text { USA }\end{array}$ & B-200-7-4 & 200 & $\mathrm{x}$ & & \\
\hline $1.27 \mathrm{~cm}$ steel rebar & Secure chamber base to soil & $\begin{array}{l}\text { Local } \\
\text { Hardware } \\
\text { Supply }\end{array}$ & & & 36 & $\mathrm{x}$ & & \\
\hline $\begin{array}{l}2.54 \mathrm{~cm} \times 6.35 \mathrm{~cm} \\
\text { U-bolt }\end{array}$ & Secure chamber base to soil & $\begin{array}{l}\text { W. W. } \\
\text { Grainger Inc. }\end{array}$ & $\begin{array}{l}\text { Lake Forest, } \\
\text { IL, USA }\end{array}$ & 1RCK8 & 27 & $\mathrm{x}$ & & \\
\hline $\begin{array}{l}0.64 \mathrm{~cm} \mathrm{LDPE} \\
\text { plastic tubing }\end{array}$ & $\begin{array}{l}\text { Connect chamber inlet and outlet to } \\
\text { the gas measurement apparatus }\end{array}$ & $\begin{array}{l}\text { United States } \\
\text { Plastic Corp. }\end{array}$ & $\begin{array}{l}\text { Lima, } \mathrm{OH} \\
\text { USA }\end{array}$ & 58097 & 708 & $\mathrm{x}$ & & \\
\hline $\begin{array}{l}\text { Assorted screws and } \\
\text { nuts/bolts for } \\
\text { chamber assembly }\end{array}$ & $\begin{array}{l}\text { Assemble chamber frame and mount } \\
\text { lid to frame }\end{array}$ & $\begin{array}{l}\text { Local } \\
\text { Hardware } \\
\text { Supply }\end{array}$ & & & 100 & $\mathrm{x}$ & & \\
\hline $\begin{array}{l}\text { Clippard Needle } \\
\text { Valves }\end{array}$ & $\begin{array}{l}\text { Control rate of chamber } \\
\text { opening/closure }\end{array}$ & $\begin{array}{l}\text { Clippard } \\
\text { Instrument } \\
\text { Laboratory } \\
\text { Inc }\end{array}$ & $\begin{array}{l}\text { Cincinnati, } \\
\text { OH, USA }\end{array}$ & JFC-2A & 342 & $\mathrm{x}$ & & \\
\hline $1 / 8 \mathrm{NPT}$ to $1 / 4$ tube & $\begin{array}{l}\text { Connect tubing to cylinder piston for } \\
\text { pneumatic chamber operation }\end{array}$ & $\begin{array}{l}\text { Swagelok } \\
\text { Company }\end{array}$ & $\begin{array}{l}\text { Solon, } \mathrm{OH} \\
\text { USA }\end{array}$ & B-400-1-2 & 110 & $\mathrm{x}$ & $\mathrm{x}$ & \\
\hline $\begin{array}{l}\text { Gast } 12 \text { VDC oil- } \\
\text { less compressor }\end{array}$ & $\begin{array}{l}\text { Pressurize air for chamber } \\
\text { opening/closure (pneumatic) }\end{array}$ & $\begin{array}{l}\text { Cole-Parmer } \\
\text { Instrument } \\
\text { Company }\end{array}$ & $\begin{array}{l}\text { Vernon Hills, } \\
\text { IL, USA }\end{array}$ & $\begin{array}{l}\text { UX-07055- } \\
60\end{array}$ & 882 & & $\mathrm{x}$ & \\
\hline $\begin{array}{l}\text { Condor MDR3 } \\
\text { compressor switch }\end{array}$ & $\begin{array}{l}\text { Turn on/shut off compressor at desired } \\
\text { pressure }\end{array}$ & $\begin{array}{l}\text { W. W. } \\
\text { Grainger Inc. }\end{array}$ & $\begin{array}{l}\text { Lake Forest, } \\
\text { IL, USA }\end{array}$ & 3FWN3 & 158 & & $\mathrm{x}$ & \\
\hline $\begin{array}{l}0.635 \mathrm{~cm} \mathrm{x} 15.24 \mathrm{~m} \\
\text { flexible copper } \\
\text { tubing }\end{array}$ & $\begin{array}{l}\text { Allow air to cool and moisture to } \\
\text { condense follow compression }\end{array}$ & $\begin{array}{l}\text { W. W. } \\
\text { Grainger Inc. }\end{array}$ & $\begin{array}{l}\text { Lake Forest, } \\
\text { IL, USA }\end{array}$ & 3Р669 & 34 & & $\mathrm{x}$ & \\
\hline $\begin{array}{l}\text { Speedaire } \\
\text { compressed air filter }\end{array}$ & $\begin{array}{l}\text { Filter compressed air and remove } \\
\text { water }\end{array}$ & $\begin{array}{l}\text { W. W. } \\
\text { Grainger Inc. }\end{array}$ & $\begin{array}{l}\text { Lake Forest, } \\
\text { IL, USA }\end{array}$ & 4ZL49 & 103 & & $\mathrm{x}$ & \\
\hline $\begin{array}{l}\text { Manifold for four- } \\
\text { channel valves }\end{array}$ & $\begin{array}{l}\text { Control pneumatic opening/closing of } \\
\text { chambers }\end{array}$ & $\begin{array}{l}\text { Clippard } \\
\text { Instrument } \\
\text { Laboratory } \\
\text { Inc }\end{array}$ & $\begin{array}{l}\text { Cincinnati, } \\
\mathrm{OH}, \mathrm{USA}\end{array}$ & $\begin{array}{l}\text { MMM-41- } \\
06\end{array}$ & 89 & & $\mathrm{x}$ & \\
\hline
\end{tabular}




\begin{tabular}{|c|c|c|c|c|c|c|c|c|}
\hline Part Name & Purpose & Supplier & $\begin{array}{l}\text { Company } \\
\text { Location }\end{array}$ & Part \# & $\begin{array}{l}\text { Total Cost } \\
\text { (for } 9 \\
\text { chambers) }\end{array}$ & Chamber & $\begin{array}{l}\text { Instrument } \\
\text { Shed }\end{array}$ & Notes \\
\hline $\begin{array}{l}\text { Four-channel, two- } \\
\text { way valves }\end{array}$ & $\begin{array}{l}\text { Control pneumatic opening/closing of } \\
\text { chambers }\end{array}$ & $\begin{array}{l}\text { Clippard } \\
\text { Instrument } \\
\text { Laboratory } \\
\text { Inc }\end{array}$ & $\begin{array}{l}\text { Cincinnati, } \\
\text { OH, USA }\end{array}$ & $\begin{array}{l}\text { MME- } \\
\text { 41PEEC- } \\
\text { W012 }\end{array}$ & 87 & & $\mathrm{x}$ & \\
\hline $\begin{array}{l}\text { Plate for extra four- } \\
\text { channel ports }\end{array}$ & $\begin{array}{l}\text { Control pneumatic opening/closing of } \\
\text { chambers }\end{array}$ & $\begin{array}{l}\text { Clippard } \\
\text { Instrument } \\
\text { Laboratory } \\
\text { Inc }\end{array}$ & $\begin{array}{l}\text { Cincinnati, } \\
\text { OH, USA }\end{array}$ & $\begin{array}{l}\text { MMM-41- } \\
\text { B }\end{array}$ & 20 & & $\mathrm{x}$ & \\
\hline $\begin{array}{l}12 \mathrm{~V}, 16 \text { channel } \\
\text { relay controller }\end{array}$ & $\begin{array}{l}\text { Control valves for gas sampling and } \\
\text { chamber operation }\end{array}$ & $\begin{array}{l}\text { Campbell } \\
\text { Scientific }\end{array}$ & $\begin{array}{l}\text { Logan, UT, } \\
\text { USA }\end{array}$ & $\begin{array}{l}\text { SDM- } \\
\text { CD16AC }\end{array}$ & 816 & & $\mathrm{x}$ & \\
\hline $\begin{array}{l}\text { Manifold for } \\
\text { Solenoid Valves }\end{array}$ & Control gas sample flow & $\begin{array}{l}\text { Clippard } \\
\text { Instrument } \\
\text { Laboratory } \\
\text { Inc }\end{array}$ & $\begin{array}{l}\text { Cincinnati, } \\
\text { OH, USA }\end{array}$ & $15781-4$ & 121 & & $\mathrm{x}$ & \\
\hline Solenoid Valves & Control gas sample flow & $\begin{array}{l}\text { Clippard } \\
\text { Instrument } \\
\text { Laboratory } \\
\text { Inc }\end{array}$ & $\begin{array}{l}\text { Cincinnati, } \\
\text { OH, USA }\end{array}$ & $\begin{array}{l}\text { DV-2M- } \\
12-\mathrm{L}\end{array}$ & 406 & & $\mathrm{x}$ & \\
\hline $\begin{array}{l}\text { Plugs for extra } \\
\text { manifold ports }\end{array}$ & Control gas sample flow & $\begin{array}{l}\text { Clippard } \\
\text { Instrument } \\
\text { Laboratory } \\
\text { Inc }\end{array}$ & $\begin{array}{l}\text { Cincinnati, } \\
\text { OH, USA }\end{array}$ & $\begin{array}{l}0035-2- \\
\text { PKG }\end{array}$ & 7 & & $\mathrm{x}$ & \\
\hline $\begin{array}{l}\text { Aalborg Mass flow } \\
\text { controllers }\end{array}$ & Control gas sample flow & $\begin{array}{l}\text { Aalborg } \\
\text { Instruments } \\
\text { and Controls }\end{array}$ & $\begin{array}{l}\text { Aalborg, } \\
\text { Denmark }\end{array}$ & $\begin{array}{l}\text { GFCS- } \\
010201\end{array}$ & 2685 & & $\mathrm{x}$ & \\
\hline $\begin{array}{l}\text { Diaphram Gas } \\
\text { Pumps }\end{array}$ & Sample inlet/outlet gas & $\begin{array}{l}\text { KNF } \\
\text { Neuberger } \\
\text { Incorporated }\end{array}$ & $\begin{array}{l}\text { Freiburg im } \\
\text { Breisgau, } \\
\text { Germany }\end{array}$ & $\begin{array}{l}\text { KNF } \\
\text { UNMP830 }\end{array}$ & 400 & & $\mathrm{x}$ & \\
\hline $\begin{array}{l}\text { 9-pin cable for MFC } \\
\text { communication }\end{array}$ & Connect MFC to datalogger & $\begin{array}{l}\text { Aalborg } \\
\text { Instruments } \\
\text { and Controls }\end{array}$ & $\begin{array}{l}\text { Aalborg, } \\
\text { Denmark }\end{array}$ & CBL-D5 & 90 & & $\mathrm{x}$ & \\
\hline Vent Needle Valves & $\begin{array}{l}\text { Control rates of overflow upstream of } \\
\text { pumps }\end{array}$ & $\begin{array}{l}\text { Clippard } \\
\text { Instrument } \\
\text { Laboratory } \\
\text { Inc }\end{array}$ & $\begin{array}{l}\text { Cincinnati, } \\
\text { OH, USA }\end{array}$ & GNV-4KI & 90 & & $\mathrm{x}$ & \\
\hline $\begin{array}{l}\text { Trojan 6V Deep } \\
\text { Cycle Battery }\end{array}$ & Power the sampling/analysis system & $\begin{array}{l}\text { Local } \\
\text { Industrial }\end{array}$ & & $\begin{array}{l}\mathrm{J} 305 \mathrm{E}-\mathrm{AC} \\
6 \mathrm{~V}\end{array}$ & 4000 & & $\mathrm{x}$ & \\
\hline
\end{tabular}




\begin{tabular}{|c|c|c|c|c|c|c|c|c|}
\hline Part Name & Purpose & Supplier & $\begin{array}{l}\text { Company } \\
\text { Location }\end{array}$ & Part \# & $\begin{array}{l}\text { Total Cost } \\
\text { (for } 9 \\
\text { chambers) }\end{array}$ & Chamber & $\begin{array}{l}\text { Instrument } \\
\text { Shed }\end{array}$ & Notes \\
\hline Solar Panels & Power the sampling/analysis system & $\begin{array}{l}\text { Battery } \\
\text { Service } \\
\text { Kyocera } \\
\text { Solar } \\
\text { Incorporated }\end{array}$ & $\begin{array}{l}\text { Scottsdale, AZ, } \\
\text { USA }\end{array}$ & $\begin{array}{l}\text { KU265- } \\
6 \mathrm{MCA}\end{array}$ & 1110 & & $\mathrm{x}$ & \\
\hline Charge Controller & $\begin{array}{l}\text { Control the charge from the solar } \\
\text { panels }\end{array}$ & $\begin{array}{l}\text { Morningstar } \\
\text { Corporation }\end{array}$ & $\begin{array}{l}\text { Newton, PA, } \\
\text { USA }\end{array}$ & $\begin{array}{l}\text { TS-MPPT- } \\
60\end{array}$ & 600 & & $\mathrm{x}$ & \\
\hline DC/AC Converter & $\begin{array}{l}\text { Convert DC battery voltage to } \mathrm{AC} \text { for } \\
\mathrm{N} 2 \mathrm{O} \text { analyzer operation }\end{array}$ & Samlex Solar & $\begin{array}{l}\text { Vancouver, } \\
\text { BC, Canada }\end{array}$ & $\begin{array}{l}\text { PST 600- } \\
24\end{array}$ & 220 & & $\mathrm{x}$ & \\
\hline $\begin{array}{l}\text { Drossbach } \\
\text { Corrugated Loom } \\
\text { Tubing }\end{array}$ & $\begin{array}{l}\text { Protect gas tubing from animal } \\
\text { damage }\end{array}$ & $\begin{array}{l}\text { W. W. } \\
\text { Grainger Inc. }\end{array}$ & $\begin{array}{l}\text { Lake Forest, } \\
\text { IL, USA }\end{array}$ & $25 \mathrm{D} 260$ & 832 & $\mathrm{x}$ & & \\
\hline $\begin{array}{l}\text { LI-COR } 830 \\
\text { CO2/H2O Vapor } \\
\text { Analyzer }\end{array}$ & Measure $\mathrm{CO} 2$ and $\mathrm{H} 2 \mathrm{O}$ & $\begin{array}{l}\text { LI-COR } \\
\text { Biosciences }\end{array}$ & $\begin{array}{l}\text { Lincoln, NE, } \\
\text { USA }\end{array}$ & LI-850 & 4400 & & $\mathrm{x}$ & \\
\hline $\begin{array}{l}\text { Teledyne } 320 \mathrm{U} \\
\mathrm{N} 2 \mathrm{O} \text { Analyzer }\end{array}$ & Measure N2O & $\begin{array}{l}\text { Teledyne } \\
\text { Technologies }\end{array}$ & $\begin{array}{l}\text { Thousand } \\
\text { Oaks, CA, } \\
\text { USA }\end{array}$ & $\mathrm{T} 320 \mathrm{u}$ & 16000 & & $\mathrm{x}$ & \\
\hline Datalogger & Measurement and control system & $\begin{array}{l}\text { Campbell } \\
\text { Scientific }\end{array}$ & $\begin{array}{l}\text { Logan, UT, } \\
\text { USA }\end{array}$ & CR3000 & 3042 & & $\mathrm{x}$ & \\
\hline
\end{tabular}

